\title{
Shaking Hands and Cooperation in Tele-present Human-Robot Negotiation
}

\author{
Chris Bevan \\ Department of Psychology \\ University of Bath \\ Bath, BA2 7AY. U.K. \\ crb23@bath.ac.uk
}

\author{
Danaë Stanton Fraser \\ Department of Psychology \\ University of Bath \\ Bath, BA2 7AY. U.K. \\ pssds@bath.ac.uk
}

\begin{abstract}
A $3 \times 2$ between subjects design examined the effect of shaking hands prior to engaging in a single issue distributive negotiation, where one negotiator performed their role telepresently through a ' $\mathrm{NaO}$ ' humanoid robot.

An additional third condition of handshaking with feedback examined the effect of augmenting the tele-present handshake with haptic and tactile feedback for the non telepresent and tele-present negotiators respectively.

Results showed that the shaking of hands prior to negotiating resulted in increased cooperation between negotiators, reflected by economic outcomes that were more mutually beneficial.

Despite the fact that the non tele-present negotiator could not see the real face of their counterpart, tele-presence did not affect the degree to which negotiators considered one another to be trustworthy, nor did it affect the degree to which negotiators self-reported as intentionally misleading one another. Negotiators in the more powerful role of buyer rated their impressions of their counterpart more positively, but only if they themselves conducted their negotiations telepresently.

Results are discussed in terms of their design implications for social tele-presence robotics.
\end{abstract}

\section{Categories and Subject Descriptors}

H.5.3 [Group and Organization Interfaces]: ComputerSupported Cooperative Work.

\section{Keywords}

Social Telepresence; Human-Robot Interaction; Cooperation; Haptic Feedback; Tactile Feedback; Social Robotics.

\section{INTRODUCTION}

Studies of interpersonal relationships have consistently shown that physical contact with other humans is an important

Permission to make digital or hard copies of part or all of this work for personal or classroom use is granted without fee provided that copies are not made or distributed for profit or commercial advantage, and that copies bear this notice and the full citation on the first page. Copyrights for third-party components of this work must be honored. For all other uses, contact the owner/author(s). Copyright is held by the author/owner(s).

HRI'15, March 2-5, 2015, Portland, Oregon, USA.

ACM 978-1-4503-2883-8/15/03.

http://dx.doi.org/10.1145/2696454.2696490. mechanism for promoting positive social interactions. Physical contact is particularly important in interactions that demand mutual cooperation and trust; so much so that it had been suggested that there can be no real trust without touch [9].

Our project "Being There: Humans and Robots in Public Spaces" is exploring how tele-presently controlled humanoid robots can allow geographically distant people to interact with physical people in public spaces. Identifying and promoting mechanisms that support mutual cooperation and trust between those people who are present within a public space and the tele-present pilot of the robot is a key goal of our research.

In a recent study of face to face interpersonal negotiation [13], the shaking of hands was found to have a strong positive effect upon cooperation - as measured by mutually beneficial economic outcomes - when negotiators shook hands prior to commencing their negotiations. In this paper, we present a study that examined whether the same effect holds when one member of a negotiating dyad conducts their role unseen, via a tele-presently controllable robot. As a tele-present negotiator is of course unable to physically touch their counterpart directly, we augmented our tele-present handshake with a virtual equivalent - haptic and tactile feedback for the non tele-present and tele-present negotiators respectively.

In concert with our primary focus on handshaking, we also sought to examine the effect of an imbalance in the communication bandwidth specific to the robot platform that we used. Our robot, the Nao (Aldebaran Robotics) has its own face. This presents a potential problem in that, when used as a tele-presence platform, interlocutors of the robot cannot see its human pilot directly without using additional hardware. As many cues to trust and cooperation are gleaned from non-verbal cues within the face (e.g. [15], [16]), conventional wisdom would suggest that a negotiator who could not see their counterpart would be at a tactical disadvantage to their counterpart who can see them. This would particularly be the case in a distributive negotiation scenario, in which the interests of both parties are mutually understood to be opposed.

\section{RELATED WORK}

The shaking of hands is a non-verbal social ritual that is practised widely in both eastern and western cultures. The use of handshaking as a symbolic gesture to signal social motives - such as willingness to cooperate and trust - is well established (e.g. [3], [4]); people shake hands on deals made; people shake hands to resolve their differences. 
The shaking of hands is a formalised form of interpersonal touch. Numerous studies of interpersonal relationships have found that brief physical contact with other humans has a positive effect upon the outcome of subsequent interpersonal interactions, providing that the touch is socially appropriate (e.g. [18], see [7] for an excellent overview). A brief touching of the shoulder of another for example can convey a sense of concern for their wellbeing, while a 'pat on the back' is commonly recognised as a signal of encouragement. Recent research by [13] found that the shaking of hands prior to engaging in a face-to-face negotiation promoted economic outcomes that were more mutually beneficial. This they argue, is because the shaking of hands serves as " $a$ subtle but critical indicator of negotiators' social motives" (p3).

This powerful effect of touch can be so subtle as to be essentially unconscious. It has been shown for instance that recipients of a brief touch to the hand promotes positive evaluations of the toucher (e.g. [6]), even though the touch itself is not explicitly remembered. Famously, in an effect referred to as the 'Midas touch', the tipping of restaurant staff was shown to increase if the staff member had briefly touched their customer's hand during the course of their service [5].

The importance of physical touch as a lubricant of social interaction has not gone unnoticed in studies of computer mediated communication (CMC) and computer-supported cooperative work (CSCW). This is for good reason. The formation of interpersonal trust and cooperation are key to the success of CSCW, yet the availability of many of the social cues upon which people rely when interacting face to face are restricted by limitations of the communication channels available to CMC (e.g. [2], [19]).

In response, a number of researchers have attempted through haptic and / or tactile feedback - to add some form of simulated touch as an additional non-verbal communication channel (e.g. [8], [1]). User studies of such technology have shown promise, finding for example that the provision of simulated interpersonal touch supports increased feelings of connectedness between people who were geographically distant from one another [17].

Replicating the social ritual of shaking hands in a computer mediated communication has been attempted using robot arms as far back as 1996 [10]. Very recently, a study by [12] found that facilitating tele-present touch through a simulated handshake enhanced positive experience of videomediated CMC.

\section{RESEARCH QUESTIONS}

Our work here seeks in part to replicate the findings of [13], and to extend their investigation to consider the effect of negotiating with an unseen tele-present counterpart. To the authors knowledge, no previous research has attempted to examine the effect of hand shaking in a tele-present humanrobot negotiation.

Reflecting and building upon the results presented by [13], our main hypotheses are summarised thus:

Hypothesis 1. Negotiations will reach a more mutually beneficial / cooperative outcome if partners shake hands prior to the negotiation than if they do not.

Hypothesis 2. Tele-present handshakes that are augmented by mutual tactile / haptic feedback (to represent the act of touch) will increase cooperative outcomes compared to handshakes without such feedback.
With regard to the inability for our robot - who represents the tele-present negotiator - to display the face of its pilot, we hypothesised that:

Hypothesis 3. Negotiators will be more willing to be intentionally misleading in their discussions with their counterparts when they perform their role tele-presently compared to when they are not tele-present.

Hypothesis 4. Negotiators will rate their counterparts as less trustworthy if their counterpart performs their role tele-presently (and thus cannot be directly seen) compared to when they are not tele-present.

Hypothesis 5. Negotiators will rate their counterpart more positively if they can see them directly compared to negotiators who cannot see their counterpart's directly.

\section{METHOD}

A controlled experiment was conducted to examine the effect upon cooperation of shaking hands prior to engaging in a single-issue distributive negotiation. Each negotiation involved two participants who were randomly assigned the role of either buyer or seller in a real-estate sale scenario. In each session, one participant performed their role in the negotiation tele-presently through a humanoid robot. The experiment was laboratory-based and used a two factor between subjects design.

The experiment had two independent variables (IV). The first IV was handshaking with three levels:

1. No handshake The negotiation was conducted without partners shaking hands at the beginning.

2. Handshake without feedback Negotiating partners are instructed to shake hands prior to commencing their negotiation.

3. Handshake with feedback As (2), but with additional feedback delivered upon touching the robot's hand: a short movement of the robot's arm and simultaneous feedback delivered to the tele-present negotiator through a short joypad vibration.

A secondary factor was also considered in that buyers could either conduct their negotiations with a tele-present seller, or vice-versa. If tele-present, the negotiator was able to both hear and see their counterpart (via a camera feed), whereas in the non tele-presence-based condition, the counterpart is represented by a humanoid robot that provided only the audio feed from the other negotiator. The second independent variable was therefore tele-presence with two levels:

1. Non-telepresent The participant performs their role in the negotiation (buyer or seller) with the other participant being represented by the robot.

2. Telepresent The participant performs their role in the negotiation (buyer or seller) tele-presently through the robot. The tele-presence system provided a live audio-visual feed from AV in the robots head, along with the ability to pan/tilt the head using a WiiMote joypad (see experimental setup).

The main dependent variable was cooperation, measured as a percentage. A value for cooperation was obtained using 
the Hamilton Real Estate negotiation task [11]. As per the experiment performed by [13], the measure of cooperation was obtained using the proportion of the potential profit captured by the buyer in a given settlement. Following this reasoning, a settlement of optimal cooperation would be $50 \%$ - a 50:50 split of the profit that was available in any given sale between the buyer and the seller.

Three secondary dependent variables were also captured using a post-negotiation questionnaire. All questions used 7 point Likert scale responses. The secondary DV's were:

1. Willingness to mislead (buyers only): The degree to which a negotiator in the buyer role self-reports as being deliberately misleading in their negotiations with the seller.

2. Trustworthiness: The degree to which negotiators in either role considered their counterpart as being trustworthy, including additional separate ratings for their perceived benevolence and honesty.

3. Impressions of the negotiating partner: The degree to which negotiators in either role rated their counterpart as likeable and pleasant to negotiate with.

\subsection{Participants}

120 participants $(\mathrm{m}=56, \mathrm{f}=64)$ were recruited from staff and students (undergraduate and postgraduate) at the University of Bath. $45 \%$ of our participants were aged between 18 and 24 years old, $43 \%$ were between 25 and 34 , and the remaining $12 \%$ were aged between 35 and 60 . Recruitment was managed through opportunity-based sampling and through advertisement on internal University mailing lists. All participants were over 18 years of age, but no other participant eligibility criteria were applied. Ethical approval for the study was sought and approved by the experimental ethics committee of the University of Bath. Participants were not rewarded for their participation.

\subsection{Apparatus and Materials}

\subsubsection{Experimental Setup}

Negotiating partners completed the experiment in separate rooms. Care was taken to prevent participants from meeting one another in person prior to their negotiation wherever possible.

The negotiation itself took place in one of the two rooms, with the physically absent negotiator conducting their assigned role (buyer or seller) tele-presently. The absent participant was represented to the other by an Aldebaran Robotics 'Nao' humanoid robot (version 4). The Nao is a $58 \mathrm{~cm}$ tall humanoid robot with 25 degrees of freedom and considerable sensory capabilities, including head mounted cameras, microphones and speakers.

Throughout the study, the robot was positioned in a neutral seated posture on a desktop, facing the participant at eye level. Internal speakers and microphones within the robot's head allowed the negotiators to speak to one another using Internet telephony. A video camera in the robot's head provided a live camera feed to the tele-present negotiator only (resolution: 320px x 240px at 30FPS). Communications were managed via a WiFi connection.

For the tele-present negotiator, the audio and video feed from the robot was presented using a laptop computer (fig.

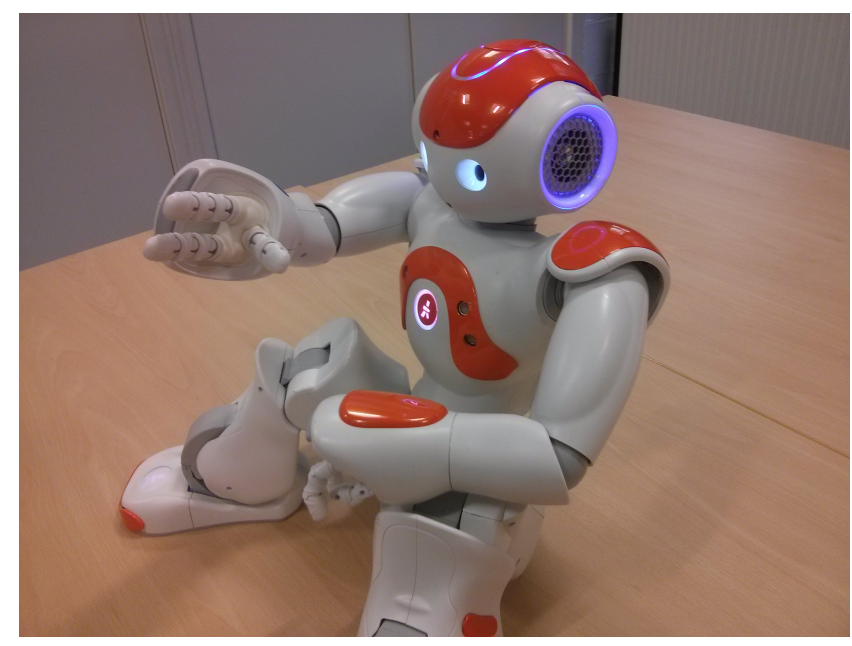

Figure 1: The handshaking gesture performed by the Nao humanoid robot.

4). A Nintendo WiiMote controller provided the ability to pan and tilt the robots head using the directional pad, and to activate / deactivate the handshake gesture through simple button press.

The handshake gesture was a custom made animation created using the Choreographe software package supplied with the Nao. When the gesture was activated, the robot extended its right arm to nearly full extension, with hand outstretched and head tilted slightly forward to ensure the hand was in view of the head mounted camera (fig. 1).

With respect to supporting a human handshake gesture, the Nao robot platform does have several limitations that are worth noting. Firstly, the shaking of hands between humans is a complex process that conducts much more information about a person than the Nao robot can realistically handle (such as grip strength and skin moisture / temperature). Secondly, the size of the Nao robot's hand is small - approximating that of a human child. While the Nao is perfectly capable of supporting the grip of an adult, its small size can make approaching the gesture slightly awkward, particularly for larger adults. Despite these limitations however, observations from pilot testing found no evidence to suggest that people had any difficulty recognising and responding appropriately to a handshake gesture performed by the Nao.

\subsubsection{The negotiation task}

The negotiation task used in the study was the two-player Hamilton Real Estate (HRE) negotiation game [11]. The HRE is an example of a single-issue distributive negotiation scenario, where the interests of the negotiating parties are mutually understood to be opposed. The materials used for the HRE were obtained from the Harvard Business Review website and were not changed in any way.

Within the negotiation, a dyad is randomly assigned the roles of buyer and seller to negotiate the sale of a piece of land. The objective of the negotiation is for a deal to be struck over the value of the land and for the sale to be made.

Both buyer and seller are provided with a private set of detailed notes that - amongst other items of strategic knowledge - outlines their respective reservation values. The difference between these reservation values is the ZOPA, or 


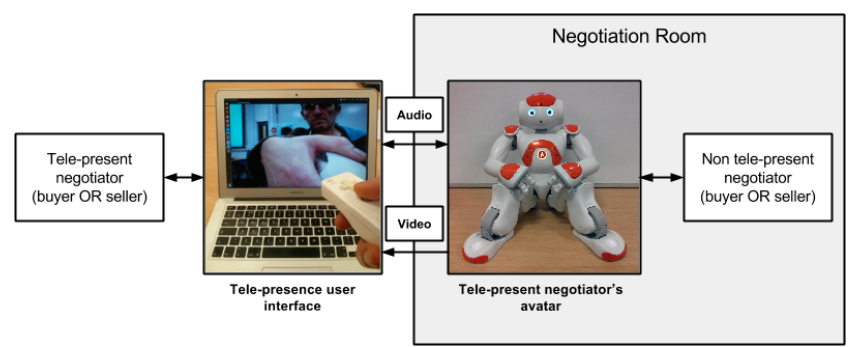

Figure 2: Study setup

zone of possible agreement. A deal can be reached only if both buyer and seller agree a price that is between these values, i.e. the sellers minimum reserve and the buyers maximum reserve.

Key to the scenario is an information asymmetry that provides a tactical advantage to the buyer should they choose to exploit it. The buyer is made aware that an imminent change in planning laws will allow them to use the land for commercial development - a significantly more profitable outcome for their company. The seller is unaware of this development, and believes that the land can legally be used for less profitable residential developments only.

\subsection{Procedure}

In each experimental session, two anonymously paired participants completed a single round of the HRE scenario from separate rooms. One participant was randomly assigned the role of the buyer, while the other assumed the role of the seller.

Upon arrival, participants were provided with a set of written instructions, after which their signed consent was obtained. Participants were informed that the experiment was not a test of their negotiation skills, and that the other negotiator was not a confederate. Both participants were then supplied with a written set of materials for their role in the negotiation and were allowed 10 minutes to prepare. Some paper and a pen was provided for them to make notes if they wished.

When ready, the tele-present participant was seated in front of the laptop, and instructions for controlling the robot were demonstrated by the experimenter. In the other room, the non tele-present participant was seated in front of the robot. The negotiation was then initiated by establishing a voIP connection (see fig. 2).

In the non handshake conditions, the participants were simply instructed to introduce themselves to one another (within role) and to begin their negotiation. In the handshaking conditions, participants were asked to introduce themselves to one another and to shake hands prior to beginning their negotiation: "it is customary in negotiations of this type to shake hands before we begin. Please shake hands".

To 'shake hands', the tele-present negotiator initiated the manoeuvre by pressing a button on the WiiMote to extend the robot's right hand. To maximise the correspondence between this action and the appearance of the robot's hand through the camera feed, the pilot was instructed to hold the WiiMote in their right hand and to point it at the screen. As the robot's right hand extended, it was clearly visible in the camera feed provided by the laptop (fig 4).

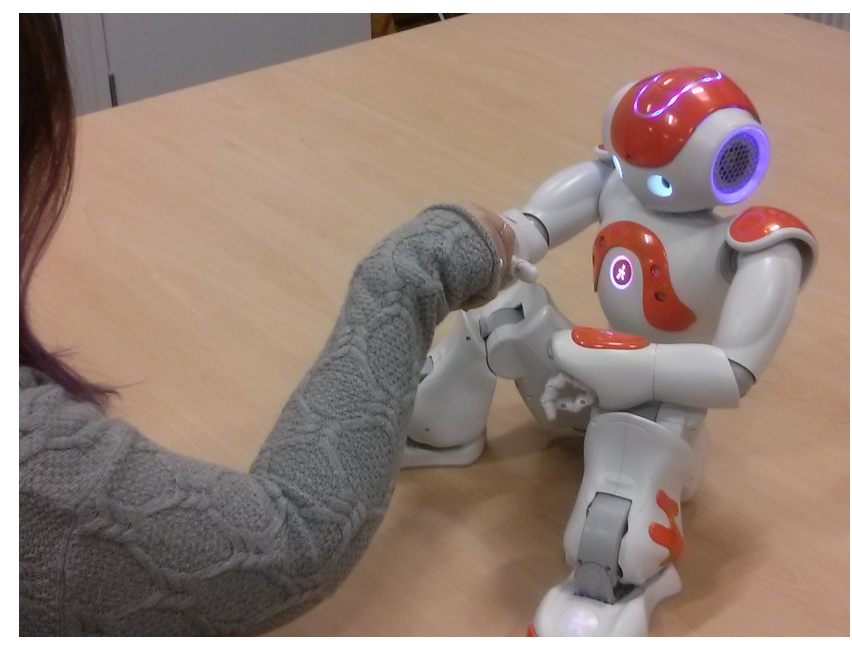

Figure 3: Shaking hands with the tele-present negotiator, as represented by a Nao robot. Grasping the robot's hand resulted in a brief automatic movement of the robot's arm up and down (see 4).

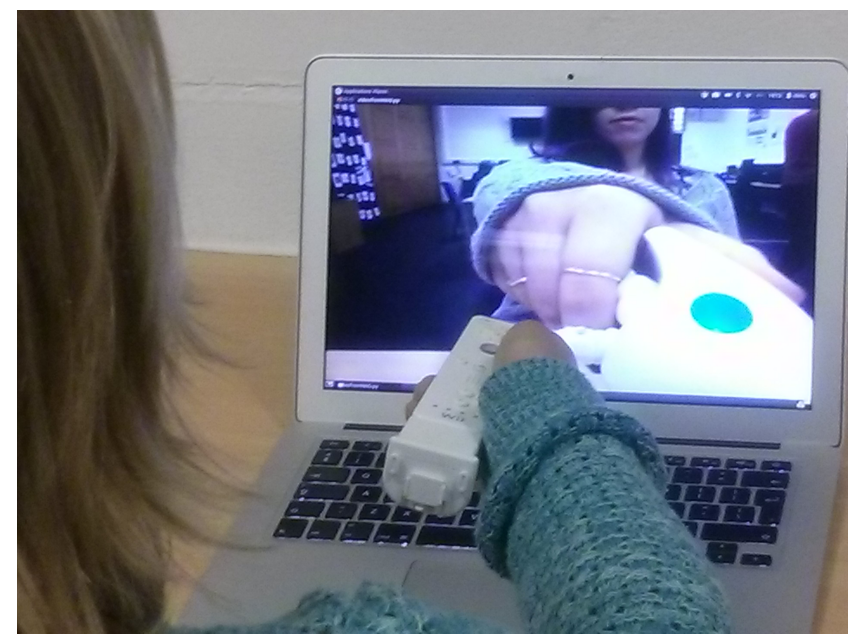

Figure 4: The tele-presence interface as seen by the robot pilot during the shaking of hands. A WiiMote is held in the right hand, corresponding to the hand extended by the robot. When the non tele-present actor grasps the robot's hand, the WiiMote's internal rumble device briefly vibrates.

In the handshake with feedback condition, as the participant grasped the robot's hand, touch sensors in the hand transmitted a signal, making the WiiMote in the tele-present participant's hand briefly vibrate. Simultaneously, the robot moved its own arm up and down several times, providing haptic feedback to the non tele-present participant (fig 3).

During the negotiation, participants were free to ask any questions of the other in order to reach a settlement figure. Following completion of the negotiation, the participants completed a short questionnaire, after which both participants were debriefed together in the same room.

The total duration of each study session was approximately 30 mins. 


\section{RESULTS}

120 participants took part in a total of 60 negotiations, with settlements ranging from $\$ 38 \mathrm{~m}$ to $\$ 66 \mathrm{~m}(M=\$ 46.6 \mathrm{~m})$. 60 participants conducted their role in the negotiation telepresently, split evenly across the roles of buyer and seller. The negotiation sessions were evenly split across the three conditions of hand shaking, with 20 instances of no handshaking, 20 with handshaking [no feedback] and 20 with handshaking [feedback].

\subsection{The effect of shaking hands and tele-presence upon cooperative outcomes in the HRE ne- gotiation scenario}

As only those negotiators who were assigned the role of buyer were in a position to exploit their tactical advantage, as per the analysis performed by [13], only the buyers $(\mathrm{N}=$ 60) were surveyed.

Cooperation scores for the buyers only were submitted to a two-way ANOVA, with handshaking entered as the first factor and tele-presence as the second factor. Two-way ANOVA revealed a statistically significant difference in cooperation scores between negotiators who shook hands and negotiators who did not shake hands prior to commencing their negotiations, $F(2,54)=11.911, p<.001$. A post-hoc Tukey test showed that the no handshake and handshake[no feedback] groups differed significantly at $p=.03$, while the no handshake and handshake[feedback] groups differed significantly at $p<.001$. Handshakes that were augmented with feedback produced the most mutually beneficial outcomes, but did not offer a statistically significant improvement over the handshake[no feedback] condition at the 0.05 level ( $p=0.07$ n.s.).

There was no statistically significant effect of tele-presence on cooperation scores, $F(1,54)=1.811, p=.184$ n.s., and there was no statistically significant interaction between handshaking and tele-presence on cooperation scores, $F(2,54)=$ $.651, p=.525$ n.s.

These results supported our first hypothesis and showed that the act of shaking hands prior to negotiating improved cooperative outcomes, regardless of whether the buyer conducted their negotiations tele-presently or not. Our second hypothesis relating to the increased benefit of providing mutual haptic feedback was only partially supported. Though it offered considerable improvement over not shaking hands, our implementation was not powerful enough to offer significantly more benefit than shaking hands without feedback.

A plot of the mean proportion of profit captured by the buyers across the three conditions of handshaking is presented in fig 5. A score of $50 \%$ is considered optimal cooperation, where the available profit in the deal is shared equally between buyer and seller.

\subsection{The effect of shaking hands and tele-presence upon willingness to mislead}

As in the previous analysis, as the role of buyer is the only role that has any utility in being intentionally misleading, only the buyers were surveyed in this analysis. A composite score for willingness to mislead was calculated for each buyer using the average of their responses to two questions (both measured using a 7-point Likert scale): 'in general, how intentionally misleading were you during the negotiation?' and 'when discussing how you intended to use the

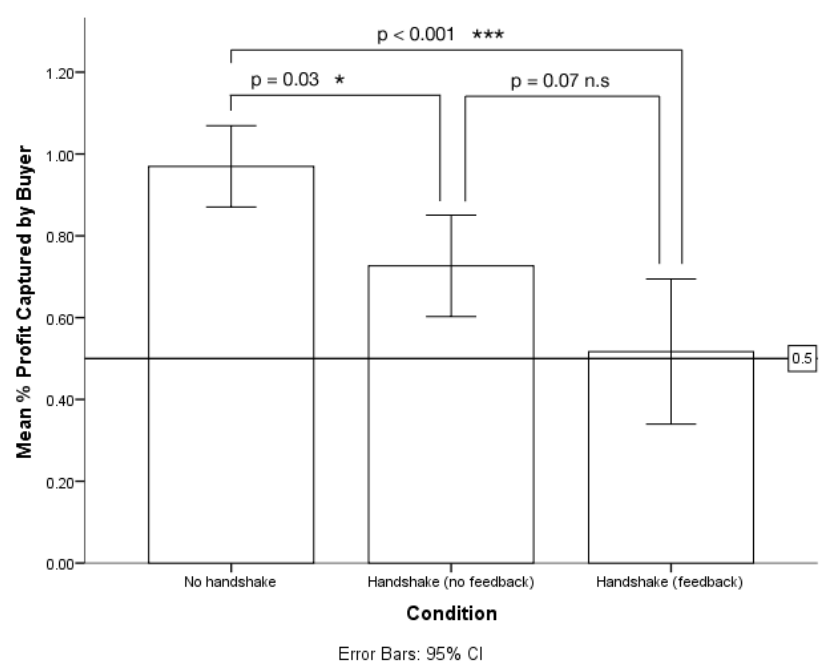

Figure 5: Mean \% profit captured by the buyers across the three conditions of handshaking. An optimum mutually beneficial outcome is $50 \%$.

land, how intentionally misleading were you?'. Both questions were rated from 1) not at all to 7) very much.

Scores for willingness to mislead for the buyers $(\mathrm{N}=60)$ only were submitted to a two-way ANOVA, with handshaking entered as the first factor and tele-presence as the second factor. Two-way ANOVA revealed no significant effect of handshaking upon willingness to mislead, $F(2,54)=0.179$, $p=0.836 \mathrm{n} . \mathrm{s}$, nor was there any statistically significant effect of tele-presence, $F(1,54)=0.007, p=0.932$ n.s. There was no statistically significant interaction between handshaking and tele-presence on willingness to mislead, $\mathrm{F}(2,54)=1.112$, $\mathrm{p}=.333$ n.s.

These results show that our third hypothesis was not supported. Neither tele-presence nor shaking hands had any effect on the degree to which buyers self-rated themselves as being intentionally misleading during their negotiations. Mean scores for willingness to mislead for both factors of handshaking and tele-presence are presented in table 1.

\subsection{Perceptions of the trustworthiness of ne- gotiating partners}

A composite score for trustworthiness was calculated for each participant as an average of their responses to three questions (all measured using a 7-point Likert scale): 'how trustworthy did you feel your negotiating partner was?', 'How benevolent / well-meaning did you feel your negotiating partner was?' and 'how honest did you feel your partner was?'. All questions were rated from 1) not at all to 7) very much. Scores for trustworthiness for both buyers $(\mathrm{N}=60)$ and sellers $(\mathrm{N}=60)$ were submitted to separate twoway ANOVAs, with handshaking entered as the first factor and tele-presence as the second factor in both cases.

For negotiators in the buyer role, two-way ANOVA revealed no significant effect of handshaking upon their impressions of the trustworthiness of sellers, $F(2,54)=0.177$, $p=0.838$ n.s. The effect of tele-presence upon trustworthiness approached significance, $F(1,54)=3.368, p=0.072$ n.s. There was no significant interaction effect between the 
handshaking and tele-presence factors, $\mathrm{F}(2,54)=0.544, \mathrm{P}$ $=.583$ n.s.

For negotiators in the seller role, two-way ANOVA revealed no significant effect of handshaking upon self reported impressions of the trustworthiness of buyers, $F(2,54)=0.747$, $p=0.478$ n.s. There was no significant effect of tele-presence upon self reported impressions of the trustworthiness of buyers, $F(1,54)=0.494, p=0.485$ n.s., and there was no interaction effect, $F(2,54)=0.016, P=.984$ n.s. Mean scores for trustworthiness across both factors of handshaking and telepresence are presented in table 1.

These results run counter to our fourth hypothesis, showing that the degree to which negotiators considered their counterpart to be trustworthy was not affected by shaking their hand or being able to see them directly.

\subsection{Impressions of negotiating partners}

A composite score for impressions of negotiating partners was calculated for each participant as an average of their responses to three questions (all measured using a 7-point Likert scale): 'what was your impression of your negotiating partner (1: very negative - 7: very positive)', 'how much did you like your negotiating partner? (1: not liked at all - 7: very much liked)' and 'how much would you like to negotiate with the same partner again? (1: not at all - 7: very much)'. As in the previous analysis, scores for impressions of counterparts for both buyers $(\mathrm{N}=60)$ and sellers $(\mathrm{N}=60)$ were submitted to separate two-way ANOVAs, with handshaking entered as the first factor and tele-presence as the second factor in both cases.

For both buyers and sellers, there was no significant effect of handshaking upon scores for the impressions of their counterparts: $F$ [buyers] $(2,54)=0.863, p=0.428$ n.s, $F$ [sellers] $(2,54)$ $=0.607, p=0.549$ n.s.

For the buyers only, there was a significant effect of telepresence in that buyers rated their seller counterparts more positively if they had conducted their own negotiations telepresently (and could thus see their seller counterpart). For the sellers, there was however no effect of tele-presence upon their impressions of the buyer, $F(1,54)=0.400, p=0.530$ n.s. No interaction effect was observed for either buyers or sellers: $F[$ buyers] $(2,54)=1.274, p=.288$ n.s., $F$ [sellers] $(2,54)$ $=0.026, P=.974$ n.s.

These results support our fifth hypothesis, but only for negotiators in the buyer role. A plot of the mean scores for impressions of the negotiating partner (both buyers and sellers) across the two conditions of tele-presence is presented in fig 6.

\section{DISCUSSION}

The results of this controlled study provides promising evidence that the ritual of shaking hands - even if the completion of this gesture is more symbolic than an accurate simulation - is an important factor in promoting cooperative social interactions using tele-presence robotics.

Our results confirmed our primary hypothesis, showing that negotiations reached a more mutually beneficial / cooperative outcome if partners shook hands prior to negotiating. This replicates the findings reported by [13], but also builds upon it by demonstrating that this effect appears to hold even in situations where a tele-present actor is represented by a humanoid robot (and as such is not directly observable by their negotiating partner). Our second hypothesis relat-

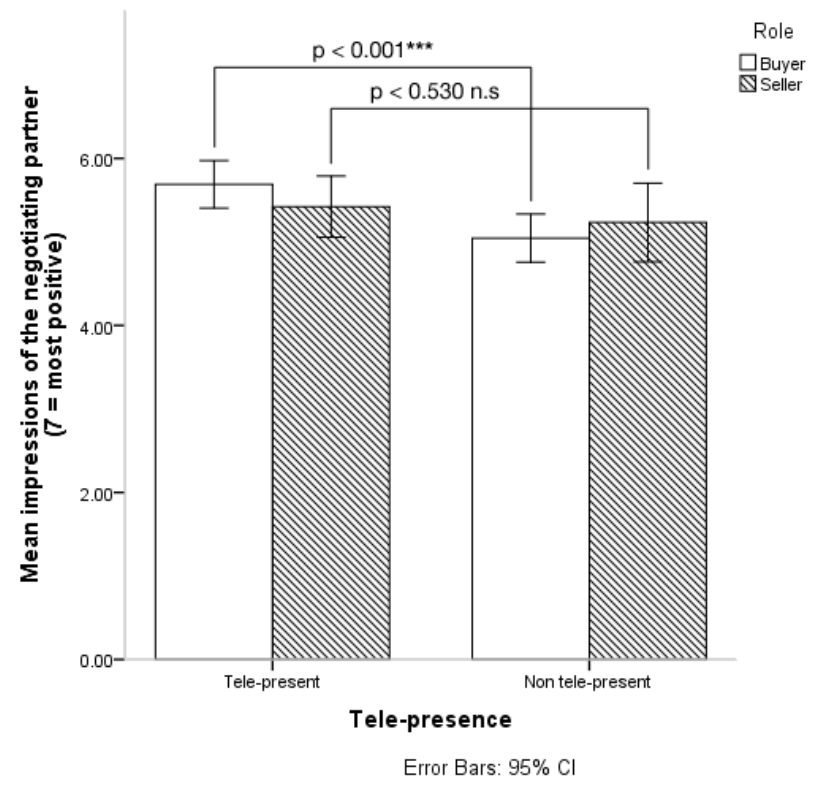

Figure 6: Mean score for impressions of the negotiating partner across tele-presence for both buyers and sellers. A higher score indicates a more positive impression.

ing to the augmentation of tele-present handshaking with mutual tactile / haptic feedback (to represent the act of touch) was also partially supported, in that the highest levels of cooperation were observed when such feedback was provided.

However, we must be cautious not to overstate our findings. Our results thus far were observed using a single robot platform that has notable physical and sensory limitations. There are potentially other mechanisms that were not explicitly addressed within the limited scope of our study that may well have a contributory influence upon the positive cooperative outcomes observed here.

In addition to our primary finding, we also found that the degree to which negotiators in the more powerful role of buyer intentionally misled their counterparts did not change if hands were shook, nor did it change when the buyer conducted their role tele-presently (such that they could observe the seller directly). This runs counter to [13]'s findings, suggesting that, even if buyers did not reveal what they knew, they still accepted a fairer settlement if hands were shook than if they did not shake hands. Also contrary to our hypothesis, our participants did not vary in their appraisal of the trustworthiness of their counterparts when hands were shook, nor did it change if the ability to see the true face of their counterpart was removed. We suggest that the unthreatening and somewhat child-like face of the Nao robot itself may be an influencing factor here, and we will explore this possibility in future studies.

Finally, our hypothesis that the negotiator would rate their counterpart more positively if they can see them directly was only partially supported, in that buyers rated sellers more positively if they themselves conducted negotiations tele-presently. Again, the appearance of the Nao 


\begin{tabular}{|c|c|c|c|c|}
\hline Role & Tele-presence & Handshaking & $\begin{array}{l}\text { Willingness to mislead } \\
{[\text { mean (SD)] }}\end{array}$ & $\begin{array}{l}\text { Perceived trustworthiness } \\
\text { of negotiating partner } \\
{[\text { mean }(\mathrm{SD})]}\end{array}$ \\
\hline \multirow{6}{*}{ buyer } & \multirow{3}{*}{ tele-present } & No handshake & $3.25(2.67)$ & $5.00(2.22)$ \\
\hline & & Handshake (no feedback) & $4.25(1.81)$ & $5.23(0.97)$ \\
\hline & & Handshake (feedback) & $4.40(2.13)$ & $5.07(0.89)$ \\
\hline & \multirow{3}{*}{ non tele-present } & No handshake & $4.25(2.76)$ & $4.53(1.17)$ \\
\hline & & Handshake (no feedback) & $4.00(1.93)$ & $4.10(1.36)$ \\
\hline & & Handshake (feedback) & $3.40(2.00)$ & $4.76(0.92)$ \\
\hline \multirow{6}{*}{ seller } & \multirow{3}{*}{ tele-present } & No handshake & $1.00(0.82)$ & $5.23(1.07)$ \\
\hline & & Handshake (no feedback) & $1.10(0.74)$ & $4.70(1.24)$ \\
\hline & & Handshake (feedback) & $1.50(0.97)$ & $5.13(5.13)$ \\
\hline & \multirow{3}{*}{ non tele-present } & No handshake & $1.20(0.95)$ & $4.90(1.37)$ \\
\hline & & Handshake (no feedback) & $1.35(0.85)$ & $4.50(1.63)$ \\
\hline & & Handshake (feedback) & $1.55(1.04)$ & $4.93(1.11)$ \\
\hline
\end{tabular}

Table 1: Mean scores for willingness to mislead and the perceived trustworthiness of the negotiating partner across all handshaking and tele-presence conditions. Scores range from 0 to 7 .

robot is potentially a contributing factor to this finding and will be explored further in future studies.

\section{DESIGN IMPLICATIONS}

In summary, though more research is required to develop and build confidence in the findings that we have presented thus far, we suggest that tele-present robotics designers should take care to ensure that their designs support handshaking by humans, and that sufficient sensors are made available to register both fleeting and sustained touches to the hand.

Several behavioural observations were also made during the execution of our study. Unprompted, a number of our participants naturally moved the WiiMote up and down during the handshake manoeuvre and were disappointed that this movement did not have any effect on their robotic avatar. Internal accelerometers in the WiiMote could feasibly support a one-to-one movement of both the robot's pilot and the robot's hand and we will explore this possibility in future refinements of this interaction. In a similar vein, our non telepresent participants were deliberately not instructed as to how to shake the robot's hand, and were instead allowed to approach this unexpected interaction intuitively. The Nao robot has only one degree of freedom in its hand, which means that the outstretching of its fingers leaves its thumb at a 90 degree angle to the wrist (see fig. 1). This sometimes rendered the grasping of its hand awkward. Though most participants were able to overcome this awkwardness and grasp the hand fully, some chose instead to merely grasp the fingers of the hand instead.

\section{LIMITATIONS AND FUTURE WORK}

There were a number of technical limitations of the study that will be considered in the design of our future studies. The first of these issues is communication latency. Though we made considerable effort to minimise the latency between grasping the robot's hand and the triggering of a vibration in the WiiMote controller held by the pilot, a latency of 0.5 sec- onds between these events was not uncommon. Researchers including [14] have reported that consistency between physical and on-screen action is critical to the interpretation of gestures and positive experience with tele-present robotics. Our findings here support this notion. Future work will explore the use of haptic devices with quicker operation times than the WiiMote, with the aim of consistently reducing this latency to imperceptible levels.

Finally, based on the experimenter's observations of the negotiations during the study, the self-reported scores we observed for 'willingness to mislead' were slightly lower than we expected. We suspect that our participants felt uncomfortable revealing the true degree to which they intentionally mislead their partners in a self report. Videotaping the negotiations was considered, and would have offered a more accurate record, but results from piloting suggested that participants would have behaved differently had they have been videotaped. This additional measure was consequently abandoned. Future studies will consider other ways to accurately record such a sensitive measure.

\section{ACKNOWLEDGMENTS}

This research grant is funded by the EPSRC under its IDEAS Factory Sandpits call on Digital Personhood, grant ref: EP / L00416X / 1. The authors would like to thank Dr. Paul Bremner (Bristol Robotics Laboratory, Bristol U.K.) for his support developing the technical apparatus used in this study.

\section{REFERENCES}

[1] J. N. Bailenson, N. Yee, S. Brave, D. Merget, and D. Koslow. Virtual interpersonal touch: expressing and recognizing emotions through haptic devices. Human-Computer Interaction, 22(3):325-353, 2007.

[2] N. Bos, J. Olson, D. Gergle, G. Olson, and Z. Wright. Effects of four computer-mediated communications channels on trust development. In Proceedings of the 
SIGCHI conference on human factors in computing systems, pages 135-140. ACM, 2002.

[3] J. K. Burgoon. Relational message interpretations of touch, conversational distance, and posture. Journal of Nonverbal Behavior, 15(4):233-259, 1991.

[4] J. K. Burgoon, D. B. Buller, and W. G. Woodall. Nonverbal communication: The unspoken dialogue. 1996.

[5] A. H. Crusco and C. G. Wetzel. The midas touch the effects of interpersonal touch on restaurant tipping. Personality and Social Psychology Bulletin, 10(4):512-517, 1984.

[6] J. D. Fisher, M. Rytting, and R. Heslin. Hands touching hands: Affective and evaluative effects of an interpersonal touch. Sociometry, pages 416-421, 1976.

[7] A. Gallace and C. Spence. The science of interpersonal touch: an overview. Neuroscience \& Biobehavioral Reviews, 34(2):246-259, 2010.

[8] A. Haans and W. IJsselsteijn. Mediated social touch: a review of current research and future directions. Virtual Reality, 9(2-3):149-159, 2006.

[9] C. Handy. Trust and the virtual organization. Harvard business review, 73(3):40, 1995.

[10] H. Hashimoto and S. Manoratkul. Tele-handshake through the internet. In Robot and Human Communication, 1996., 5th IEEE International Workshop on, pages 90-95. IEEE, 1996.

[11] D. Malhotra. Hamilton real estate (tn). Harvard Business School Teaching Note, pages 910-037, 2010.

[12] H. Nakanishi, K. Tanaka, and Y. Wada. Remote handshaking: touch enhances video-mediated social telepresence. In Proceedings of the 32nd annual ACM conference on Human factors in computing systems, pages 2143-2152. ACM, 2014.

[13] J. Schroeder, J. Risen, and F. Gino. Handshaking Promotes Cooperative Dealmaking. Available at http://dx.doi.org/10.2139/ssrn.2443674, 2014.

[14] D. Sirkin and W. Ju. Consistency in physical and on-screen action improves perceptions of telepresence robots. In HRI '12: Proceedings of the seventh annual ACM/IEEE international conference on Human-Robot Interaction, pages 57-64, 2012.

[15] M. Stirrat and D. I. Perrett. Valid facial cues to cooperation and trust male facial width and trustworthiness. Psychological science, 21(3):349-354, 2010.

[16] M. van 't Wout and A. G. Sanfey. Friend or foe: The effect of implicit trustworthiness judgments in social decision-making. Cognition, 108(3):796-803, 2008.

[17] R. Wang, F. Quek, D. Tatar, K. S. Teh, and A. Cheok. Keep in touch: channel, expectation and experience. In Proceedings of the SIGCHI Conference on Human Factors in Computing Systems, pages 139-148. ACM, 2012.

[18] F. N. Willis Jr and H. K. Hamm. The use of interpersonal touch in securing compliance. Journal of Nonverbal Behavior, 5(1):49-55, 1980.

[19] J. Zheng, E. Veinott, N. Bos, J. S. Olson, and G. M. Olson. Trust without touch: jumpstarting long-distance trust with initial social activities. In $C H I$ '02: Proceedings of the SIGCHI Conference on Human Factors in Computing Systems. ACM, 2002. 